\title{
DECISIONAL INTEROPERABILITY: CONCEPTS AND FORMALISATION
}

\author{
Daclin N., Chen D., Vallespir B. \\ LAPS, University Bordeaux 1, ENSEIRB, UMR 3151,351 cours de la Libération, 33405 \\ Talence cedex, FRANCE, nicolas daclin@laps.u-bordeaux1.fr
}

\begin{abstract}
This paper deals with interoperability of enterprises. Interoperability is a multi-form issue and can be studied at different levels. This research work focuses on the business level and particularly on the decisional aspect so called decisional interoperability. The objective of the paper is to present how to develop decisional interoperability using design principles with mathematical formalisation, in order to improve and to facilitate the decisionmaking activity in a collaborative context. Finally, an illustration example using the proposed principles is presented to show the interest of the decisional interoperability solution.
\end{abstract}

\section{INTRODUCTION}

Increasingly, competitiveness of an enterprise depends not only on its internal productivity and performance, but also its ability to collaborate with others. Enterprises need to collaborate with other partners in terms of communication and interaction, in order to reach their objectives including the ones that are common between partners. In this context the development of interoperability has become a key factor of success.

Interoperability can be studied at different levels, and one of the developments of interoperability is related to the decision-making activity (ATHENA, 2003). It is socalled decisional interoperability. The purpose of this paper is to present the basic concepts and principles of decisional interoperability.

However, this paper does not take into account the socio-psychological aspect of the decision-making but focuses on formal and quantitative aspects, in order to reduce the uncertainty in terms of the objectives that the partners have to reach during a collaborative decision-making process.

This work has been initiated within the frame of WP6 (Design principles for interoperability) of INTEROP NoE (INTEROP, 2003). It also aims at extending the GRAI decisional model concepts to the development of interoperability in the context of networked enterprises.

First, the definition and a mathematical formalisation related to the decisionmaking activity inside one enterprise are presented. Set theory is used to formalise the decision-making activity.

Then, the decision-making activity in a collaborative context is discussed. Based on these works, a definition of decisional interoperability is proposed. Some design principles to build a decisional interoperability solution are presented. Set theory 
notations are also used to formalise the basic concepts of the decisional interoperability.

A simple case study is proposed at the end of the paper in order to illustrate and show the applicability of the proposed approach.

\section{DECISION-MAKING ACTIVITY: CONCEPTS AND DEFINITIONS}

\subsection{Definition}

Decision-making is an activity that aims at making choice. The decision itself is the result of choosing between courses of action (CEN, 2000). The decision-maker disposes of a set of alternatives and has to choose the best one allowing reaching his objectives. According to (Doumeingts, 1998) and (Vallespir, 2002), in order to choose among possible solutions, several items may influence the decision-maker:

- One (or more) objective(s) that the decision must allow reaching;

- One (or more) decision variable(s) on which the decision-maker can act;

- Constraints that limit the use of the decision variables; and

- Sometimes, criteria that guide the choice to make during the decisionmaking process.

This set of items form a decision frame constraining the degree of freedom to make a decision. Therefore, the decision-making can be shown as the search of a solution reaching (or being near) the objectives in a space defined by the decision variables and delimited by the constraints (Vallespir, 2003). Figure 1 illustrates the principle of decision-making consistently with the decision frame.

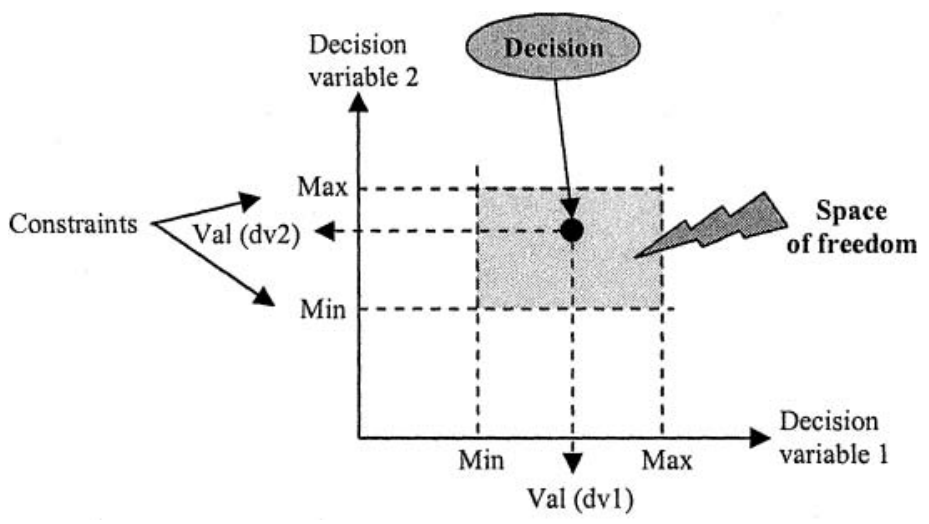

Figure 1-Representation of the space of freedom for decision-making (two decision variables are considered) 


\subsection{Formalisation}

Starting from the definitions given above, it is possible to propose a formalisation of the decision-making activity. Formalisation is necessary to understand and represent in a precise way the basic concepts relating to decision-making activity. Furthermore, it will allow giving general rules to make a decision that could be applicable in any situation including decisional interoperability.

Let us assume several notations:

Given $O B$ the set of objectives that the decision must allow reaching,

$$
O B=\left\{o b_{1}, \ldots, o b_{n}\right\}, n \in \mathrm{N}^{*} \text {. }
$$

Given $V D$ the set of decision variables,

$$
V D=\left\{v d_{1}, \ldots, v d_{n}\right\}, n \in \mathrm{N} *
$$

Given $V D_{c t}$ the set of constrained decision variables,

$$
V D_{c t}=\{v d \in V D \mid P(v d)\}=\left\{v d_{1 c 1,1}, . v d_{n c t u}\right\}, n \in \mathrm{N}^{*},
$$

with $P(v d)=$ the decision variables respect the constraints.

In order to make the decision through the formalisation of the decision frame and the definition given by (Vallespir, 2003), it is possible to write:

Given $D$ the set of the possible decisions (given by the set of the values of the decision variables defined by the constraints),

$$
\begin{gathered}
D=\left\{\left\{v d_{1_{c t 1}, \ldots,}, v d_{n c m}\right\}\right\}, d=\left\{v d_{1 c 1,}, \ldots v d_{n c t i n}\right\}, n \in \mathrm{N}^{*} \\
\operatorname{card}(D)=\operatorname{card}\left(v d_{1 c 1}\right) \times \ldots \times \operatorname{card}\left(v d_{n c t m}\right), n \in \mathrm{N}^{*}
\end{gathered}
$$

To each element of $D$ - that is to say each combination of values of the decision variables - one possible objective can be associated.

Given $O B^{\prime}$ the set of objectives that can be reached by the elements of $D$,

$$
O B^{\prime}=\left\{o b^{\prime} \in O B^{\prime} \mid o b^{\prime}=f(d)\right\} .
$$

The function $f$ defines a series of mathematical operation that allow the transformation of the decision variables values into the corresponding objectives. An objective, belonging to $O B$, can be totally fulfilled if and only if it also belongs to $O B^{\prime}$. In the case where there is no solution, the decision-maker will choose a solution belonging to $O B^{\prime}$ that will be the nearest to the desired objective.

Finally, the set $D_{\mu}$ of the decisions researched $d$ is:

$$
D r=\left\{d \in D \mid O B^{\prime} \cap O B\right\} \text {. }
$$

This formalisation can be now applied to decisional interoperability. 


\section{DECISIONAL INTEROPERABILITY}

\subsection{Collaborative decision-making}

In the context of collaboration, partners work together in order to reach a common goal (Kvan, 2000). At the level of decision-making, it concerns the decision-makers to take a decision reaching the objective of the collaboration and also satisfying their own interests. Currently, the decision-making process is well defined (Mercier, 2003). However, several problems emerge from this process (Chen, 2005) (Wikipedia, 2006):

- The majority of collaborative decisions have to do with subjective issues (there is no formalisation and no rational manner to take a decision);

- Decisions are usually imposed by one party to another;

- The time length to achieve a common decision.

Therefore, decisional interoperability has to support the collaborative decisionmaking activity and to prevent the mentioned problems.

\subsection{Decisional interoperability}

According to (IEEE, 1990), (Oxford, 1999) and (Daclin, 2005), decisional interoperability can be defined as the ability for several decision-makers to exchange support-information for decision-making, and to use these support-information in order to make a decision that allows reaching the objective of the collaboration and at the same time that respects their own interests. The principle is to generate a common decision space taking their own support-information for decision-making into account as shown in figure 2 .

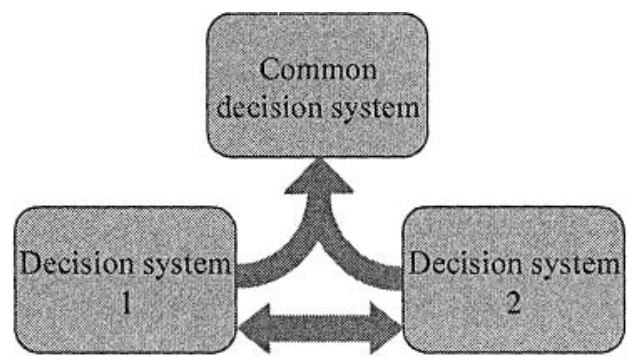

Figure 2 - Representation of the principle of decisional interoperability (Daclin, 2006)

The existing collaborative decision-making process is not designed for decisional interoperability. Consequently, it is important to define principles to design decisional interoperability solutions.

\subsection{Design principles for decisional interoperability}

A design principle is a rule to follow, that orients design decision-making. It can be defined as a fundamental truth which stands for evidence (Suh, 1990). Starting from 
the problems encountered in collaborative decision-making, three design principles for decisional interoperability have to be implemented according to the identified problems as summarised in figure 3 .

In the case 1, decision-maker A makes a decision without knowing neither decision frame nor the possible decision which can be made by decision-maker B. In this case, the decision-maker A can make a decision which, on the one hand does not satisfy the decision-maker $\mathrm{B}$ and on the other hand does not satisfy the objective of the collaboration. This leads to some iterations and negotiations between both decision-makers to find a solution.

In the second case (case 2) the decision-makers A and B have clearly defined their decision frame including the possible decisions that can be engaged and make it known to the partner. In this case, the decision-makers can make a decision on the one hand without time loss and on the other hand which can satisfy both of them and the objective of the collaboration.

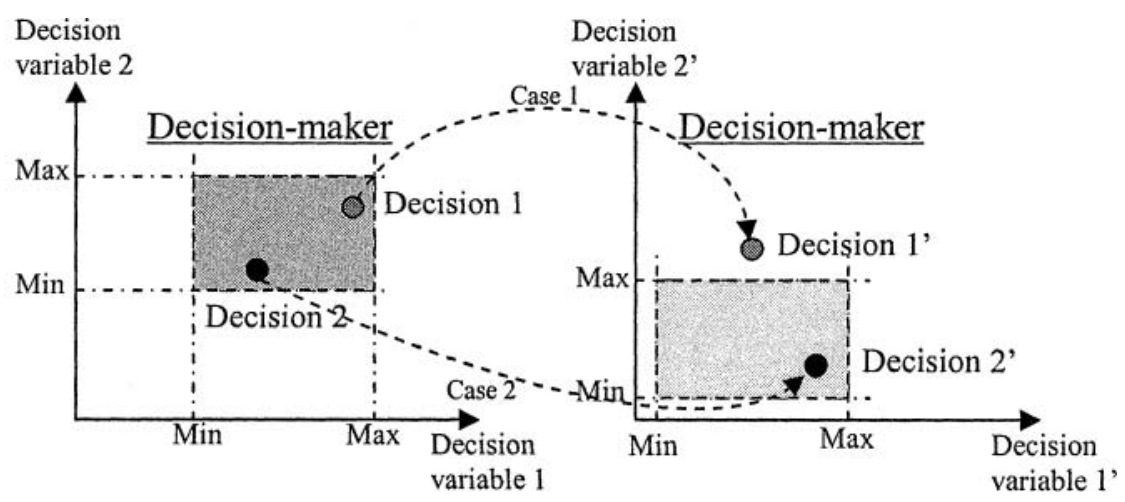

- Bad decision of $\mathrm{A}$ leading to a decision of $\mathrm{B}$ out of its frame

- Good decision taken by $\mathrm{A}$ knowing the decision frame and the capability of $\mathrm{B}$

Figure 3-Representation of the problems of collaborative decision-making in order to establish design principles

The three design principles for decisional interoperability that have to be implemented are:

Axiom 1: "when designing a decision-making activity, make its decision frame explicit".

The decision frame contains a set of items which limits the freedom of decisionmaking. It allows improving the decision-making transparency.

Axiom 2: "Implement a mechanism to exchange the decision frame between all decision-makers".

Building a decision frame known by your collaborative decision-maker improves decisional interoperability in terms of iterations between parties, and of the delay before reaching a decision. 
Axiom 3: "Establish capability for each decision-maker".

Decisional capability allows knowing admissible limits for the decision-makers, and improves the ability to make decisions that satisfy all decision-makers as far as possible.

\subsection{Formalisation}

Based on the conceptualisation of the decision-making activity previously proposed, a formalisation of the decisional interoperability is developed below.

Let us assume supplementary notations:

Given $O B_{c}$ the set of the objectives of the collaboration,

$$
O B_{c}=\left\{o b_{c 1}, \ldots, o b_{c n}\right\}, n \in \mathrm{N}^{*} .
$$

Given $P_{c}$ the set of the possible decisions that can be engaged by each partner in the collaboration,

$$
P_{c}=\left\{p_{c 1, \ldots,} p_{c n}\right\}, n \in \mathrm{N}^{*} .
$$

In order to obtain this set, the decision-makers have to exchange their own decision frame as defined above; otherwise they risk making a decision that will not allow satisfying other partners and/or the objective of the collaboration. As a consequence, the partners will have more negotiations and iterations to make a satisfying decision. This set represents the capability of the decisions-makers.

Given $D_{c}$ the set of the possible decisions in the frame of the collaboration,

$$
\begin{gathered}
D_{c}=\left\{\left\{p_{c 1}, \ldots, p_{c n}\right\}\right\}, d_{c}=\left\{p_{c 1}, \ldots, p_{c n}\right\}, n \in \mathrm{N}^{*}, \\
\operatorname{card}\left(D_{c}\right)=\operatorname{card}\left(p_{c 1}\right) \times \ldots \times \operatorname{card}\left(p_{c n}\right), n \in \mathrm{N}^{*} .
\end{gathered}
$$

Given $O B^{\prime}{ }_{c}$ the set of objectives that can be reached by the elements of $\mathrm{D}_{\mathrm{c}}$,

$$
O B^{\prime}{ }_{c}=\left\{o b^{\prime}{ }_{c} \in O B^{\prime}{ }_{c} \mid O b^{\prime} c=g\left(d_{c}\right)\right\} \text {. }
$$

The function $g$ defines a series of mathematical operation that allow the transformation of the possible decisions that can be engaged by each partner into the corresponding objectives that can be reached in the frame of the collaboration.

An objective, belonging to $O B_{c}$, can be totally fulfilled if and only if it belongs to $O B^{\prime}{ }^{\prime}$.

Finally, the set $D_{r c}$ of the decisions researched $d_{c}$ is:

$$
D_{r c}=\left\{d_{c} \in D_{c} \mid O B^{\prime}{ }_{c} \cap O B_{C}\right\}
$$

This formalisation is applied to an illustration example presented in the section 4 . This example is largely simplified to illustrate the decisional interoperability between two partners using a formal approach. 


\section{ILLUSTRATION EXAMPLE}

Let us consider two partners $\left(\mathrm{P}_{\mathrm{c} 1}\right.$ and $\left.\mathrm{P}_{\mathrm{c} 2}\right)$, in a collaborative decision-making context.

The objective of the collaboration for the partners is: "to produce n products for a maximum cost of $30 \mathrm{~K} \epsilon$ ". This objective can be noted:

$$
O B_{c}=\{0,1, \ldots, 30\}
$$

The partners have selected the possible decisions (in this case in euro) that can be engaged in the frame of the collaboration. This selection of the possible decisions is performed and workable after that the decision-makers have clearly defined and exchanged their own decision frames. According to their own decision frame and the decision frame of the partner, these possible decisions can be noted:

$$
\begin{aligned}
& P_{c 1}=\{10,15,17\} \\
& P_{c 2}=\{13,16,24\}
\end{aligned}
$$

The set of possible decisions of the collaboration is noted $D_{c}$.

$$
\begin{aligned}
& \operatorname{card}(D c)=\operatorname{card}(P c 1) \times \operatorname{card}(P c 2) \\
& \operatorname{card}(D c)=3 \times 3=9
\end{aligned}
$$

Thus, there are 9 elements in $D c$. It is possible to write them on:

$$
D c=\left\{\begin{array}{l}
\{10,13\},\{10,16\},\{10,24\} \\
\{15,13\},\{15,16\},\{15,24\} \\
\{17,13\},\{17,16\},\{17,24\}
\end{array}\right\} .
$$

The set of objectives that can be reached by the elements of $\mathrm{D} c$ is $O B^{\prime}{ }_{c}$.

It has been written above that $o b^{\prime} c=g(d c)$. In this example, the objective is a cost and the cost supported by the partners must be summed. Therefore, in this case, the $g$ function is defined by the sum of all elements of a given $d_{c}$ set:

$$
o b^{\prime} c=\sum_{i=1}^{n} d c i
$$

Thus, for the set of the objectives that can be reached by the elements of Dc, it gets:

$$
O B^{\prime} c=\{23,26,34,28,31,39,30,33,41\} .
$$

Finally, the set of the decisions researched by the collaborative decision-makers can be noted:

$$
\text { cost } \leq 30 \Rightarrow \text { Drc }=\{\{10,13\},\{10,16\},\{15,13\},\{17,13\}\} .
$$

Any of these four possibilities fulfils the given objective. Indeed, they meet the objective of the collaboration defined as "to produce n products for a maximum cost of $30 \mathrm{KE}$ ". The decision-makers can make a decision among the $D_{r c}$ set of solutions. 


\section{CONCLUDING REMARKS}

This paper has presented a formalisation that aims at developing interoperability in the context of collaborative decision-making in order to solve and to prevent the problems identified (iterations, long delay...). This formalisation is independent from technological issues.

Some design principles to build decisional interoperability were proposed in order to allow the decision-makers to make right and appropriate decisions by clearly defining their decision frame, exchanging their decision frame and establishing a common decision space. This formalisation is simple to design and implement in enterprises and can apply in any kind of situations. The example is largely simplified to keep the presentation in a reasonable volume.

The proposed approach allows extending the GRAI decisional model concepts initially developed for intra enterprise integration to inter enterprise interoperability from the point of view of decision-making.

Future work is concerned with the development of functions - that allow obtaining the set of objectives that can be reached by the possible decisions of the partners of the collaboration - based on performance criteria of the enterprises. These functions represent the performances that are the most frequently targeted by the enterprises such as cost, quality and time. A real case study will also carried out in a SME.

\section{REFERENCES}

1. ATHENA. Advanced Technologies for Heterogeneous Enterprise Networks and their Applications, FP6-2002-IST-1, Integrated Project Proposal, 2003.

2. CEN TS 14818. Enterprise integration - Decisional Reference Model, CEN TC310/WG1, 2000.

3. Chen, David. "Practices, principles and patterns for interoperability". INTEROP deliverable D6.1, 2005.

4. Daclin N, Chen D, Vallespir B. "Design principles and pattern for decisional interoperability". In proceedings of IFIP 5.7 Advances in Production Management Systems, APMS, 2005.

5. Daclin N, Chen D, Vallespir B. "Towards a conceptualisation of decisional interoperability", Interoperability of Enterprises and Software Applications, I-ESA'06, 2006.

6. Doumeingts $G$, Vallespir B, Chen D. "Decisional modeling GRAI grid". In International handbook on information system, P. Bernus, K. Mertins \& G. Schmidt ed, Berlin: Springer, 1998.

7. IEEE. "A compilation of IEEE standard computer glossaries", standard computer dictionary, 1990.

8. INTEROP. "Annex1-Description of work", Interoperability Research for Networked Enterprises Applications and Software. INTEROP NoE. Network of Excellence, $n^{\circ} 508011,2003$.

9. Kvan, Thomas. "Collaborative design: what is it?". In Automation in Construction, vol.9, Elsevier Science BV, 2000.

10. Mercier N, Noyes D, Clermont P. "Modélisation des mécanismes de collaboration dans les processus décisionnels". 4ème congrès des doctorants, 2003.

11. Oxford dictionary. "Oxford dictionary", Oxford University Press, 1999.

12. Suh NP. "The principles of design", Oxford University Press, ed, 1990.

13. Vallespir B, Doumeingts G. "La méthode GRAI". Support de cours de l'école de printemps "modélisation d'entreprise", groupe de travail $\mathrm{n}^{\circ} 5$ du groupement pour la recherche en productique, 2002.

14. Vallespir, Bruno. "Modélisation d'entreprise et architectures de conduite des systèmes de production". Mémoire d'Habilitation à Diriger des Recherches, 2003.

15. Wikipédia. "Les systèmes de prise de decision, www.wikipédia.fr, 2005. 